\title{
Electrochemical Behavior and Determination of four drugs using Multi-Wall Carbon Nanotubes Modified Glassy Carbon Electrode
}

\author{
Xia Zhao ${ }^{1}$, Yan Zhang ${ }^{1}$, Dingfeng Gao ${ }^{1}$, Huabin Xiong ${ }^{1,2,3, *}$, Yuntao Gao ${ }^{1,2,3}$, \\ Simin $\mathrm{Li}^{1}$,Xiaofen Li ${ }^{1,2}$, Zhi Yang ${ }^{1,2}$, Manhong Liu ${ }^{1,2}$, Jianhui Dai ${ }^{1,2}$, Donghua Zhang ${ }^{1,2}$ \\ ${ }^{1}$ School of Chemistry and Environment, Yunnan Minzu University, Kunming 650500, China. \\ ${ }^{2}$ Key Laboratory of Comprehensive Utilization of Mineral Resources in Ethnic Regions, Yunnan \\ Minzu University, Kunming 650500, China. \\ ${ }^{3}$ Center International Joint Research of Clean Use of Biomass Resources in Cross-border Ethnic \\ Regions Yunnan, Yunnan Minzu University, Kunming 650500, China. \\ *E-mail: yunxhb@126.com
}

doi: $10.20964 / 2019.01 .44$

Received: 6 September 2018 / Accepted: 28 October 2018 / Published: 30 November 2018

\begin{abstract}
A glassy carbon electrode (GCE) that is modified with a thin film of multi-wall carbon nanotubes (MWCNT) is a useful tool for determining concentration, and a new method for obtaining this type of electrode with excellent determination characteristics is presented. In this paper, the electrochemical behavior of picoplatin, caffeic acid, ferulic acid, and breviscapine on the electrode was studied by cyclic voltammetry and showed effective improvements in terms of the sensitivity of measurement. The optimum conditions for the determination were investigated. Differential pulse voltammetry was used to determine the content of four drugs in the actual sample. The electrochemical detection method based on MWCNTs/GCE has a low detection limit, good recovery, and precision in pharmaceuticals determination. Therefore, the method is simple, rapid, accurate, and reliable to apply in pharmaceuticals field.
\end{abstract}

Keywords: MWCNTs/GCE; Pharmaceuticals determination; Electrochemical Behavior; Detection limit

\section{FULL TEXT}

(C) 2019 The Authors. Published by ESG (www.electrochemsci.org). This article is an open access article distributed under the terms and conditions of the Creative Commons Attribution license (http://creativecommons.org/licenses/by/4.0/). 